\title{
LAS FORMAS DEL TURISMO CIENTÍFICO EN AYSÉN, CHILE.
}

\author{
Fabien Bourlon \\ Departamento de Turismo Sustentable \\ Centro de Investigación en Ecosistemas de la Patagonia (CIEP) \\ Universidad Austral de Chile. \\ fabienbourlon@ciep.cl \\ Pascal Mao \\ Institut de Géographie Alpine \\ Université Joseph Fourier - Grenoble I \\ Francia. \\ pascal.mao@ujf-grenoble.fr \\ mao.cermosem@gmail.com
}

\section{RESUMEN}

El presente artículo, ideado en el marco del proyecto "Las Ciencias al servicio del desarrollo turístico de Aysén, creando un Centro para el Turismo Científico de la Patagonia”, llevado desde 2007 por el Centro de Investigación en Ecosistemas (CIEP), trata de la noción del turismo científico y sus formas en la Patagonia chilena. Basado en una extensa revisión literaria se propondrán cuatro formas específicas de prácticas o productos turísticos, asociados a diferentes enfoques y en diversos grados, a la dimensión científica. Estas formas son: 1. El turismo de exploración y de aventura con una dimensión científica, 2. El turismo cultural con contenido científico, tanto aquel cercano al ecoturismo como al turismo industrial, 3. El eco-voluntariado científico y 4. El turismo de investigación científica. Para cada forma mostraremos cómo la puesta en marcha de proyectos en Aysén ha llevado a un análisis reflexivo y a explicitar el Turismo Científico allí. Posteriormente se discutirán las condiciones de aparición de la noción a nivel internacional, su pertinencia en las dinámicas del turismo contemporáneo y sus expresiones en la Patagonia Chilena. Al no ser evidente, para muchos autores, el vínculo entre ciencia y turismo, esta contribución propondrá debates y, en algunos casos, puntos de vista opuestos.

Palabras claves: turismo científico, revisión literaria, investigación, Patagonia, Aysén. 


\title{
FORMS OF SCIENTIFIC TOURISM IN AYSÉN, CHILEAN PATAGONIA.
}

\author{
Fabien Bourlon \\ Departamento de Turismo Sustentable \\ Centro de Investigación en Ecosistemas de la Patagonia (CIEP) \\ Universidad Austral de Chile. \\ fabienbourlon@ciep.cl \\ Pascal Mao \\ Institut de Géographie Alpine \\ Université Joseph Fourier - Grenoble I \\ Francia. \\ pascal.mao@ujf-grenoble.fr \\ mao.cermosem@gmail.com
}

\begin{abstract}
The present paper, conceived within the project "Sciences supporting tourism development of Aysén, creating a Centre for Scientific Tourism for Patagonia”, carried out since 2007 by the Research Center on Patagonian Ecosystems (CIEP), refers to the notion of scientific tourism and it's formes in Chilean Patagonia. Based on an extensive literature revue, it offers to distinguish four specific forms of tourism practices and / or products, linking in a variety of ways and degrees, the scientific dimension. These forms are 1. Explorations and adventure tourism with scientific dimensions, 2. Cultural tourism with scientific contents similar to either ecotourism or industrial tourism, 3. Scientific eco-volunteering and 4. Scientific research tourism. For each forms we will show how the implementation of projects in Aysén imposed an extensive analysis and specification of Scientific Tourism there. We than discuss the conditions for appearance of the notion world wide, its relevancy within present tourism dynamics and its expression in Chilean Patagonia. The interrelation between science and tourism not being an obvious one for numerous authors, this contribution puts forward debates and sometimes opposed views.
\end{abstract}

Key words: scientific tourism, literature review, research, Patagonia, Aysen. 


\section{Las formas del turismo científico en Aysén, Patagonia Chilena}

En el marco del proyecto "Las Ciencias al servicio del desarrollo turístico de Aysén, creando un Centro para el Turismo Científico de la Patagonia”, llevado desde 2007 por el Centro de Investigación en Ecosistemas (CIEP), se estudia la noción del turismo científico y sus formas en la Patagonia chilena (cf. www.turismocientifico.cl ).

Como una necesaria introducción al concepto de turismo consideramos que las diversas características asociadas al termino "turismo" pueden clasificarse en tres grandes grupos (Dewailly, 2006). El primero guarda relación con la dimensión espacial del fenómeno. Turismo de montaña, rural, litoral, urbano, verde, de naturaleza, son algunas de las expresiones que permiten especificar geográficamente el área de extensión de las prácticas relacionadas. El segundo integra una dimensión ética como el turismo sustentable, responsable o solidario. Finalmente, el tercer grupo como el turismo deportivo, de aventura, cultural, de negocios, educativo, apunta a especificar los tipos y formas de prácticas sociales relacionadas. Numerosos autores lamentan la multiplicación de los términos utilizados en el campo del turismo y la abundancia de epítetos (MIT, 2002, Stock, 2003). Cazes (1998) define algunos de estos como "palabrería o palabras ambiguas", "calificativos de adorno” o incluso "vocablos superfluos”. En este contexto, ¿cómo definir un "turismo deportivo sustentable de montaña" cuando cada uno de sus calificativos ha dado lugar a una profusión de debates relacionados con sus adecuaciones y limitaciones respectivas? podemos citar entre otros a Debarbieux (2001) en el caso de la montaña, a Lamic (2008) en cuanto a la capacidad del turismo para ser sustentable o Bourdeau (2006) para los deportes en áreas alpinas.

Sin embargo, el uso de calificativos tiene el mérito de intentar diferenciar y fijar una denominación de los fenómenos socioespaciales para los menos heteróclitos que engloba actualmente el campo de observación del turismo. Siguiendo esta perspectiva, diversas publicaciones buscan definir y circunscribir algunas nociones como el turismo de aventura (Buckley, 2010) o turismo deportivo (Bouchet, Lebrun, 2009) e identificar sus diferentes dominios de aplicación. Estas obras permiten realizar una evaluación necesaria de la situación y, sobre todo, proponen una definición que posteriormente se pueda discutir y enmendar. Siguiendo esta lógica, la presente contribución apunta a definir y cuestionar el uso del término "turismo científico". Esta última aparece frecuentemente desde mediados de la década de los 90 en la literatura científica, técnica o entre operadores del sector turístico. 
En la primera parte, la revisión literaria permitirá identificar los diferentes tipos y formas de transferencia de la dimensión científica en el sector turístico. Se identificaron, definieron y detallaron cuatro formas principales de turismo científico. En esta parte no se pretende hacer referencia a todos los contribuyentes académicos a nivel internacional que usan el término turismo científico, sino que mostrar la diversidad de los usos posibles de esta noción y sus dominios de aplicación. De hecho, asociar el turismo y los dominios científicos puede parecer a priori contradictorio. A través de la reflexión generada en el marco del proyecto "Las Ciencias al servicio del desarrollo turístico de Aysén, creando un Centro para el Turismo Científico de la Patagonia”, se profundizará el análisis mostrando cómo fue necesario para el caso una reflexión crítica en la aplicación del concepto. De esta forma, se presentarán debates y puntos de vista defendidos por diferentes autores. La segunda parte permitirá discutir las condiciones de aparición de la noción y su pertinencia en las dinámicas del turismo contemporáneo, tal como en el caso en un territorio específico como Aysén.

\section{Las cuatro formas de turismo científico}

De manera estrictamente informativa ${ }^{1}$, se realizó una búsqueda de palabras clave en "Google académico" que permitió identificar 620 referencias en español y portugués que utilizaban los términos "turismo científico", "turismo de investigación” o "turismo de pesquisa”, 94 en francés ("tourisme scientifique” o "tourisme de recherche”), 51 en alemán (“wissenschaftourismus") y, finalmente, más de 3000 en inglés ("scientific tourism” o "research tourism”). A pesar de las diversas limitantes inherentes a este tipo de recuento, este último muestra el uso compartido de la noción de turismo científico, particularmente en la literatura en español, portugués e inglés. Sin embargo, una lectura más profunda de numerosas obras, artículos, informes de ingeniería, de estudio y de investigación muestra que la noción de turismo científico se utiliza para calificar una gran variedad de prácticas o de productos turísticos. Algunos privilegian áreas de aventura o de exploración, culturales, educativas o vivenciales o la esfera de la investigación científica stricto sensu. Las cuatro formas que se presentan a continuación constituyen, por lo tanto, una visión que permite distinguir las principales esferas de uso de la noción.

\footnotetext{
${ }^{1}$ Búsqueda realizada el 21 de marzo de 2011. Todas las contribuciones identificadas no tratan completamente sobre el turismo científico (búsqueda de palabras clave). Además, los resúmenes han sido traducidos en varios idiomas, lo que favorece el conteo múltiple de una gran cantidad de publicaciones como la multireferencia de algunas en diversos sitios de internet. Tal vez, no se trata de simples citas bibliográficas. Por lo tanto, estos valores están muy sobrevalorados y se muestran aquí sólo con propósitos indicativos. Además, es importante destacar que un gran número de referencias bibliográficas presentes en esta contribución no son referencias en los motores de búsqueda (publicaciones documentos de artículos, de informes de proyectos).
} 


\section{a).- El turismo aventura de dimensión científica}

Esta primera forma de turismo científico permite asociar las dimensiones científicas a las prácticas de exploración, de aventura o deportivas. La investigación científica puede tener un lugar variable en la motivación del desplazamiento turístico: simple excusa, pretexto o justificación o, al contrario, puede constituir la motivación principal de la práctica (en este último caso, esta forma se acerca más al turismo de investigación científica que se detalla a continuación).

Los mundos del turismo de naturaleza o de montaña y de investigación científica han estado estrechamente vinculados a lo largo de su historia. Morse (1997) los considera incluso como componentes indisociables en la exploración de nuevos territorios durante el siglo XIX. El caso de John Muir ilustra perfectamente este vínculo. Él es considerado como uno de los alpinistas pioneros de Sierra Nevada (Chirs, 1997; Selters, 2004) que publicó las primeras descripciones de montaña después de la conquista de diversas cumbres (The Mountains of California en 1894 y My First Summer in the Sierra en 1911; obras agrupadas en Muir, 1992). Apasionado por la botánica y la geología, Muir fue un autodidacta a pesar de haber tomado algunos cursos en la Universidad de Wisconsin; no obtuvo su título porque privilegió la escuela de la aventura y de los viajes para perfeccionar su educación (Gretel, 2000) de manera similar al Tour de los gentlemen ingleses en Europa un siglo antes. Él adquiere progresivamente legitimidad académica a través de la publicación de diversas contribuciones. Así, su renombre contemporáneo se basa tanto en sus múltiples exploraciones como en su influencia en las ciencias del medioambiente (Hall, 2010; Miller, Morrison, 2005).

Esta interconexión entre turismo aventura y ciencia se va institucionalizando progresivamente y perdurará hasta el periodo contemporáneo. Un ejemplo de Europa es la Société des Explorateurs Français (SEF) creada en 1937, adquiere desde sus orígenes el patrocinio de la sociedad de geografía de París que acoge a la asociación en sus locales. La SEF y el Groupe Liotard, creado en 1945 en el Musée de l’Homme, fijan como misión conjunta "reunir a las personas deseosas y susceptibles de participar en misiones científicas, artísticas o deportivas, en el descubrimiento del mundo (sitio de la societe-explorateurs.org)”. Clot (2009), quien reconstituye 100 años de exploración francesa de los miembros de la SEF, muestra a través de aproximadamente 70 expediciones importantes cómo la dimensión científica está presente casi sistemáticamente de forma paralela al descubrimiento y la exploración de nuevos territorios. Bajo el mismo modelo existen numerosas sociedades a lo largo de todo el mundo; por ejemplo, la Scientific Exploration Society de Reino Unido fundada en 1969, la Exploration Society of Southern Africa (1988) o incluso la Israel Exploration Society (1914). 
Esta relación se encuentra también en otras organizaciones menos elitistas. Así, El Club Alpin Français (CAF) siguiendo el modelo del Club Alpino Británico o del Club Alpino Suizo (Hoibian, Defrance, 2002) integró rápidamente, después de su creación en 1874, la dimensión científica a sus actividades (primero la topografía, y posteriormente la astronomía, aerología y meteorología, geografía humana, zoología o botánica a través de la formación de comisiones y consejos científicos (Hoibian 2000a). Este objetivo perdura hasta el día de hoy en la asociación: "la vocación generalista del CAF, además de privilegiar las prácticas relacionadas con el alpinismo, integra el conjunto de dominios relacionados con la montaña: las ciencias, la cultura y el medioambiente" (estatutos del comité científico del CAF, 2010)

De la misma forma, la Unión Internacional de Espeleología (UIS, por sus siglas en inglés) implementó un código "para la Exploración de Cavidades e Investigación Científica en el Extranjero" cuya función es fomentar iniciativas que permitan "descubrir nuevas cavidades y aumentar la exploración de las cavidades ya conocidas; estudiar su contenido (por ejemplo: minerales y restos biológicos, arqueológicos y antropológicos); difundir en el mundo los conocimientos relativos a karst y cavidades, etc." (Sitio de la U.I.S.: uis-speleo.org). Este posicionamiento que yuxtapone el estudio de medioambientes con las prácticas recreativas se encuentra en numerosas actividades deportivas. Las diferentes prácticas de buceo están reagrupadas, por ejemplo, en la Fédération Française d’Etudes et de Sports Sous-Marins y en la Nautical Archaeology Society (Reino Unido).

Para expresar esta forma de Turismo Científico en Aysén el caso del expedicionario Cristián Donoso, en 2006, es emblemático. En su proyecto para el concurso Rolex justifica su expedición en kayak por los fiordos de Aysén por la necesidad de identificar sitios arqueológicos, de naufragios (como el de la Fragata Wager), de muestreos biológicos o geológicos, lo que le permite ganar el premio. Sin embargo al cabo de esta expedición el material o las publicaciones científicas producidas son escasos, solo aportando antecedentes que motivan posteriores expediciones científicas. Otro caso es la exploración aventurera deportiva de la Scientific Exploration Society a la isla Wager en 2006. La lideraron ex buzos de la armada británica para identificar posibles restos del naufragio famoso de John Byron. En este caso no los acompañaban arqueólogos ni se trabaja en base a metodologías científicas y la motivación principal fue de corte cultural o deportivo. Sin embargo, nuevamente, dio pié, posteriormente, a investigaciones propiamente científicas por arqueólogos chilenos.

En 2011 podemos destacar el caso del Departamento de Geografía de la Pontificia Universidad Católica de Santiago de Chile, que realiza una exploración de los fiordos de Bahía Exploradores hasta 
la Laguna San Rafael en kayak, con el fin de conocer el territorio para instalar a futuro una base de investigación. No se observan investigaciones en estricto rigor en el proceso.

A modo de conclusión podemos ver que los participantes durante las expediciones, exploraciones o actividades presentan, bajo diferentes formas, justificaciones, pretextos o valores añadidos científicos, además de las hazañas puramente deportivas o recreativas. Así, todas las actividades deportivas y todos los dominios científicos pueden estar involucrados. La importancia de la investigación científica depende de la actividad deportiva: puede ser un simple pretexto que facilite la obtención de patrocinadores o la mediatización de la realización; o a la inversa, el objetivo central de la expedición (o uno de ellos). Por ejemplo, alpinistas que asocian a la ascensión de una cumbre, la medición altimétrica de esta última, o donde los espeleólogos desarrollan trabajos de hidrokarstología durante el descubrimiento de una nueva red.

\section{b).- El turismo cultural de contenido científico}

Esta segunda forma corresponde a un turismo cultural y patrimonial que presenta un contexto científico de mediación, animación e interpretación. La dimensión científica es una parte de la oferta, lo que la distingue de los productos turísticos clásicos. Las nociones de cultura y de patrimonio poseen aquí una definición extensa que integra los ambientes naturales y sociales y las dimensiones históricas y territoriales.

Esta definición es similar a la propuesta por el comité regional de turismo científico de Saguenay-Lac-Saint-Jean (CSL Sagueney - Lac St Jean, 2005) sobre el Turismo de Aprendizaje Científico (TAS, por sus siglas en francés) que se diferencia del Turismo de Investigación Científica (TRS), que se presenta a continuación en la tercera forma denominada eco-voluntariado científico. El TAS “consiste en viajes educativos organizados, estructurados y elaborados en torno a un tema científico. Un grupo restringido de turistas, deseosos por aprender y experimentar, realiza las actividades propuestas. [...] los viajes educativos son guiados por especialistas del área que comparten su experticia y permiten a los turistas aprender mientras viven una experiencia única”. Hora y Cavalcanti (2003) hablan de "turismo pedagógico" a través de la mediación científica. Esta última suele aplicarse en dos dominios diferentes: el turismo industrial y el ecoturismo.

En Europa, particularmente en Francia, la ciencia se moviliza frecuentemente para valorizar desde un punto de vista turístico los espacios del patrimonio industrial. Orget du Cluzeau (2005) asocia el turismo científico a la visita de sitios “ciencias, técnicas e industrias” (STI), tal como el 
observatorio Météo France de Mont-Aigoual, el horno solar de Odeillo, la turbina mareomotriz de la Rance o los planetarios (Belaën, 2007). Este se transforma de hecho en una derivación del turismo cultural a pesar de las diversas divergencias que persisten en términos de público y de oferta (Origet du Cluzeau, 2005, Cousin, 1998 y 2001). En una jornada de estudio, organizada en Dijon, Francia en 2004 titulada “Turismo científico - Turismo cultural, y descubrimiento de las ciencias y técnicas”, se fijó entre los objetivos definir: “¿cuáles son los vínculos entre cultura, ciencia y turismo? ¿Cómo se define el turismo científico, técnico e industrial?”. Esta expresión del turismo científico, técnico e industrial sería producto de un informe elaborado por Malécot en 1981 (citado en Cousin, 2000) que trata sobre la cultura científica, técnica e industrial.

En otros lugares como en América del Norte, la dimensión científica se aplica particularmente a la mediación de espacios naturales y de sus ecosistemas, pero también puede extenderse a sitios o ambientes de interés arqueológico, histórico o etnológico. En este caso, el turismo científico es muy parecido a la noción de ecoturismo del que puede ser un derivado. Mieczkowski (1995) define el turismo científico como "una forma de ecoturismo que ayuda a preservar un medioambiente relativamente inalterado”. Rovinsky (1993) muestra cómo esta forma de turismo de dimensión científica, participa en la preservación de espacios naturales públicos y privados en Costa Rica. Siguiendo esta misma lógica, Breton (2004) ve el "turismo científico y cultural de descubrimiento, de exploración y de tiempo libre” como uno de los paradigmas fundadores del ecoturismo, ya que "respeta las exigencias o es compatible con las obligaciones ecológicas, tal como están definidas y sancionadas por las normas jurídicas de protección y de conservación del medioambiente en su acepción patrimonial extensiva”. Se puede observar una multiplicación de los productos de operadores turísticos, tanto desde el punto de vista de las agencias especializadas como de las generalistas que proponen viajes científicos o ecoturísticos en sus catálogos. Grandes agencias u organizaciones de investigación van hasta el punto de etiquetar a algunos operadores o productos, a semejanza de la alianza de cooperación firmada en 2008 entre el CNRS de Francia y el operador turístico Escursia. Los investigadores del CNRS participan en la dirección y la presentación de los viajes. Puede tratarse del descubrimiento del bosque amazónico de Guayana Francesa, etnias del sur de Camerún o de la botánica de Madagascar (ofertas de viajes científicos, Escursia.fr, 2011). Otros autores (Morse, 1997) asocian también esta forma de viajes educativos o de estudio ("learning travel”) en el contexto de escuelas o universidades (incluso reencuentros de ex-alumnos). Este tipo de viaje está muy desarrollado en Norteamérica y forma parte de los cursos obligatorios o electivos de muchos estudiantes. En América del Sur, se utilizan comúnmente las denominaciones de "turismo científico/ educativo" y de "turismo científico y didáctico”. El Ministerio do Turismo (2010) de Brasil utiliza indistintamente los términos "turismo de universidad, turismo para la educación, turismo científico y 
turismo de estudiantes". El Ministerio de Turismo (2010) define este concepto como "turismo de estudio y de intercambio, basado en la promoción de las actividades y programas de aprendizaje que puedan favorecer el desarrollo personal y profesional gracias a experiencias interculturales”.

En la región de Aysén, observamos la puesta en marcha del turismo científico cultural cuando se generan viajes con mayor contenido y temática científico-cultural. Podemos destacar así la implementación de nuevos Circuitos en la Reserva Nacional Coyhaique, donde los administradores (CONAF) aprueban la incorporación de mayores contenidos, a través de guías de campo, en este caso relacionadas con la gestión forestal. En esta misma línea, la publicación del libro "Arnold Heim, un Geólogo Suizo en la Cuenca del Lago General Carrera”, por Staub y Barrientos, en 2009, genera hoy el contenido para un viaje temático al sector.

En el ámbito de lo que hemos descrito como "Learning Travel”, el viaje de estudio realizado por la Universidad de Montana en enero 2011, basado en el ámbito de los impactos medio-ambientales de posibles represas hidroeléctricas, vemos cómo un tema construye un viaje educativo. Al contrario del viaje realizado por la Universidad de Michigan y que describiremos más adelante este viaje no da lugar a publicaciones o aportes científicos nuevos. Se trata de un viaje educativo y cultural, que participa de la educación de los alumnos.

Circuitos llevados por la Estancia Punta del Monte, asociado a la temática de los cóndores y de la presencia indígena tehuelche, permite entender dos temas regionales muy relevantes. Acompañado del dueño, el visitante puede hacer diversas observaciones ornitológicas y arqueológicas.

Como podemos apreciar el turismo cultural de contenido científico es un segmento amplio en sus expresiones temáticas y sus formas de implementación. Permite a viajeros aventureros descubrir un territorio o la realización de viajes más convencionales siguiendo el hilo conductor de un relato de la cultura local. Es seguramente en este ámbito que podríamos observar el desarrollo de un turismo más masivo.

\section{c).- El eco-voluntariado científico}

Esta tercera forma es cercana al "ecoturismo científico" presentado anteriormente, pero agrega una participación directa y activa del turista / voluntario en la construcción y el desarrollo de la actividad de investigación científica. Guiado por los investigadores, el voluntario se transforma en un actor participante de la ejecución del protocolo metodológico de la investigación, en la recolección de 
datos o de información, incluso está asociado al tratamiento y valorización de la actividad científica. (Wearing, Neil, 2000; Wearing, 2001). El ecovoluntariado integra una dimensión ecológica o medioambiental a la noción de voluntariado, de esta forma, el eco-voluntariado designa una acción voluntaria al servicio de la protección o valorización de las especies y hábitats naturales, de la preservación del medioambiente, de proyectos humanitarios o de desarrollo social (Blangy, Laurent, 2007; Baillet, Berge, 2009). De hecho, el ecoturismo, basado en la observación, la interpretación y la educación medioambiental difiere del eco-voluntariado, ya que este último implica obligatoriamente acciones que se deben realizar y tareas que se deben cumplir en un proyecto colectivo. "La inversión del visitante es muy variable y va desde un simple viaje de descubrimiento y de comprensión [calificado op.cit. de ecoturismo] hasta un viaje de eco-voluntariado donde el viajero se compromete en cuerpo y alma en el proyecto de desarrollo, de conservación o de investigación científica (Blangy, Laurent, 2007).

En este caso, esta forma se acerca al Turismo de Investigación Científica (TRS) propuesto por el comité regional de turismo científico de Saguenay-Lac-Saint-Jean (op.cit., CSL Sagueney - Lac St Jean, 2005). Este turismo se define como "una actividad de tiempo libre especializada en ciencias puras y aplicadas. Esta actividad es practicada por turistas deseosos de conocer el proceso de investigación científica y participar del avance de la ciencia. La duración de la actividad deber ser bastante prolongada para permitir una formación, una integración adecuada y el cumplimiento de objetivos precisos. El turismo científico implica la supervisión de actividades científicas estructuradas por un investigador o experto. Además, incluye obligatoriamente una formación que apunta a recolectar datos siguiendo el método científico” (CSL Sagueney - Lac St Jean, 2005).

En Aysén destacamos algunos ejemplos con el propósito de explicitar el concepto en cuanto a viajes educativos y culturales. Aquí el turista es, de manera activa, participe de un proceso. El caso de eco-voluntariado con un objeto científico, es el viaje de Estudio de la Universidad de Michigan realizado en 2008 en relación al tema de los impactos ambientales de las posibles represas hidroeléctricas. Estudiantes y profesores producen desde el trabajo en terreno, encuestas y entrevistas, un análisis prospectivo del tema, realizando así un aporte a la comunidad y a la academia. Existe un informe escrito relatando el estudio.

En si el viaje más emblemático de Eco-Voluntariado Científico, es sin lugar a dudas el de investigaciones arqueológicas en el valle Chacabuco dirigido por los científicos Francisco Mena (CIEP) y Ana Prentiss (Universidad de Montana) en 2011. En este caso voluntarios pagan para ser parte del grupo de trabajo de investigación, cubriendo así los gastos asociados a su visita y contribuyendo con su pago en un $40 \%$ del costo total de la expedición científica. 
Podemos destacar también que el científico puede ser no solo un conductor del proceso (como en este caso anterior) pero también un facilitador para que los actores locales co-desarrollen la investigación, como en el caso de los estudios históricos y pre-históricos dirigidos por el antropólogo Mauricio Osorio en el sector denominado Cerro Galera, comuna de Coyhaique. Con el Comité Campesino y el apoyo de estudiantes universitarios, un proyecto de investigación se pone en marcha. Es un caso de "acción participativa" donde los voluntarios no pagan pero aportan con sus conocimientos y donde el centro de investigación y la comunidad cubren los gastos operativos.

Existen así diversas formulas que en particular implican una participación financiera variable del voluntario: desde el pago total por parte del organismo de acogida de la estadía o del viaje hasta la compra de un servicio turístico clásico. Se proponen diversas ofertas que van desde trabajos arqueológicos hasta la participación en la protección de especies o de espacios naturales (Ellis, 2003) e incluso estadías en laboratorios y la participación en programas de investigación (Wearing, 2001 ; Stebbins, Graham, 2004). Grandes operadores turísticos comienzan a interesarse en este sector. Así la guía turística francófona "Petit Futé” promociona el eco-voluntariado en varias de sus guías temáticas tituladas, "el turismo solidario”, “la guía del ecoturismo”, “101 viajes insólitos”, que presentan numerosos viajes de contenido científico.

\section{d).- Turismo de investigación científica}

El turismo de investigación o de expedición científica, involucra directamente a investigadores que viajan por razones de trabajo o de experimentación a terreno, por colaboraciones o intercambios internacionales o reuniones, congresos, seminarios o coloquios. Así, este turismo es similar al turismo de negocios. Es importante destacar que algunos autores anglófonos utilizan una sutileza léxica que permite distinguir "scientific tourism" que corresponde a las 3 formas desarrolladas anteriormente y “research tourism”que corresponde a este último (Benson, 2004; Novelli 2004). En alemán, se utiliza el término "wissenschaftourismus".

Dar esta definición impone ciertas precauciones de uso al momento de calificar e integrar el campo turístico con la actividad científica de un investigador y sus desplazamientos profesionales. Esta idea genera debates tanto en la literatura académica francófona como anglófona. Los autores defienden (a veces duramente) tres puntos de vista principales (Dewailly, 2006).

El primero apunta a excluir del análisis del hecho turístico los desplazamientos profesionales y, por ende, científicos. "Hablar de turismo de negocios es absurdo” (MIT, 2002, véase también 
Simmons, Leiper, 1993; Leiper, 1995; Stock, 2003, Knafou, Violier, 2005). Esta postura tiende a seguir la de Lévi-Strauss que fue ampliamente mediatizada por la frase inaugural de Tristes Trópicos (1995): “Odio los viajes y a los exploradores”, que apunta a distinguir al investigador para quien el viaje es un medio y no una finalidad, con el objetivo de adquirir datos e informaciones y, así, construir conocimiento académico.

El segundo, defendido por Hoerner (2002) apunta a distinguir que "un hombre de negocios [y por lo tanto un investigador] es un turista en su hotel, en el restaurant o en cualquier otro momento en que se distrae después de la cena. No lo es cuando ejerce sus funciones”. Esta definición confiere al investigador una personalidad dual al momento de realizar viajes profesionales cuya motivación principal de movilidad y su estatus durante ésta son difíciles de discernir. Este punto de vista tiene el mérito, por otra parte, de reconocer, al menos parcialmente, el vínculo entre movilidad de investigadores y el fenómeno turístico.

El tercero, que nos interesa aquí, ya que sin este la cuarta forma de turismo científico no existiría, sigue la definición clásica dada por la Organización Mundial de Turismo que estipula "el turismo es un desplazamiento fuera del lugar de residencia habitual por más de 24 horas, pero menos de 4 meses, por razones de ocio, objetivos profesionales (turismo de negocios) o un objetivo sanitario (turismo sanitario)”. Diversos investigadores están contentos con esta definición. “" We do not believe that pleasure tourism can be studied in isolation of others forms of tourism such as business travel » (Shaw, Williams, 2002).

Desde esta perspectiva, durante sus viajes a las zonas polares, Hall et al. (Hall, 1992; Hall, Johnston, 1995 et Hall, Saarinen, 2010) asocian las estadías exclusivamente recreativas y de descubrimiento a aquellas realizadas por otros motivos (investigadores, pero también empelados de bases polares o militares). Hall et al. (op. cit.) observan todos estos usos de manera indistinta y constituyen para ellos el fenómeno turístico de los destinos polares en su conjunto. Todos tendrían las mismas formas de consumo de los lugares (Urry, 1995). En sus obras sobre la Geografía Turística de Costa Rica, Vargas Ulate (1997) define a los "turistas científicos como profesionales de las ciencias sociales o naturales que realizan trabajos de investigación activos y participativos en espacios protegidos”. Esta postura permite superar el “¿choque imposible o impensable?” entre el investigador y su objeto de estudio en un destino lejano (noción relativa) deseado por Michel (1998), en este caso particular, para él, el antropólogo y el turista (esta idea no me queda muy claro). Thurner (1999) defiende la misma tesis cuestionando el vínculo entre las motivaciones de los investigadores de las ciencias sociales y sus terrenos de observación. 
González Rodríguez (2004) define el turismo científico como un complemento del ecoturismo. Él muestra el interés de los Parques Nacionales Colombianos por atraer investigadores motivados por "el aprendizaje, la experimentación y el descubrimiento, gracias a la ciencia y a los viajes en ambientes naturales donde pueden realizar sus investigaciones”. El estudio del turismo relacionado con las investigaciones científicas en Costa Rica de Laarman y Perdue (1989) da varias luces. Ellos muestran cómo los científicos han participado con sus estudios y han fomentado la construcción de un destino naturalista (creación de conocimiento y de infraestructura) que da lugar posteriormente a la creación de viajes ecoturísticos. La encuesta realizada a investigadores muestra una alta frecuencia de viajes después de sus investigaciones en terreno y su influencia en la movilización de otras personas que visitan el país (familia, amigos, estudiantes, redes sociales). Para Vargas Ulate (1997) y Pauchard (2000) el turismo científico en Costa Rica adquiere una gran importancia a partir de los años 80, debido a la afluencia de investigadores que realizan investigaciones en parques nacionales y reservas biológicas y forestales del país. Siguiendo esta misma perspectiva, Wilkie y Carpenter (1999) ilustran cómo la investigación científica participa en la protección y en el desarrollo de los espacios naturales de África Central. En los espacios periféricos, este tipo de turismo, a largo plazo, puede levantarse por medio de su estructuración en los territorios turísticos emergentes.

En el territorio de Aysén presentamos a continuación tres expresiones de esta forma de Turismo Científico vinculado expresamente a las investigaciones de los ecosistemas y/o el territorio. Una exploración Científica en el ámbito de la arqueología, para identificar la presencia pre-histórica Tehuelche, llevada por Francisco Mena (CIEP) en el Valle Chacabuco en 2009 constituye un buen ejemplo de un viaje que genera datos claves para plantear una investigación con fondos específicos posterior. A continuación de esta exploración diversos programas educativos, culturales y de ecovoluntariado han surgido?

Otro caso son las investigaciones científicas específicas donde los investigadores requieren de logística y servicios turísticos para producir datos importantes para el conocimiento y la valoración cultural de Aysén. En caso de los estudios sobre el naufragio de la Fragata Wager, llevado por el arqueólogo Diego Carabias y sus colegas (de la Universidad del Mar, de la Universidad Austral de Chile y de México) en 2011. Ellos requieren de mucha logística, transporte, alojamiento, hospedaje y servicios de guías, que consiguen gracias al apoyo de operadores locales de turismo. Con los resultados de sus campañas de investigación, producen publicaciones científicas y materiales educativos. 
Otro buen ejemplo es el trabajo vinculado a estudios acústicos y fotográficos de cetáceos en el litoral norte de la región, liderado por Heike Vester de Ocean Sounds y el Max Planck Institute en colaboración con el CIEP en 2010, produce innovadores datos sobre presencia de cetáceos y niveles de ruido en los fiordos. Hoy da lugar a un producto de turismo científico, ofrecido por la empresa, spin off de CIEP, Exploraysen SA, lo que permitirá recibir voluntarios y co-financiar la continuidad del trabajo.

Como vemos los casos son múltiples, a veces asociado a investigaciones universitarias regionales (CIEP), nacionales (como las Universidades del Mar, Concepción, Austral, PUC, de Chile, etc...) e internacionales.

\section{El turismo científico ¿ Un nuevo nicho turístico?}

A pesar de que los mundos del turismo y de la investigación puedan parecer a priori antinómicos, estas cuatro formas muestran las distintas maneras como se moviliza la dimensión científica en el sector turístico. La discusión, por consiguiente, puede tratar sobre la legitimidad de esta asociación y su inscripción en las tendencias consolidadas que inciden en las relaciones de nuestras sociedades en los viajes y en las movilidades.

El turismo científico se centra en la experiencia más que en el consumo turístico

Uno de los puntos comunes entre las diversas formas de turismo científico es la búsqueda de sentido y de justificación durante la movilidad turística. Viajar sí, pero de manera útil como lo propone el portal del eco-voluntariado (www.eco-volontaire.com ). Idea que también podemos encontrar en la obra de Stebbins y Graham (2004), “Volunteering as leisure / leisure as volunteering”. Esta noción de ser útil es tanto para ayudar a otros, participación en un proyecto colectivo, como para uno mismo, en términos de educación. El CSL Sagueney - Lac St Jean (op.cit., 2005) integra de esta forma el turismo científico en un "turismo de aprendizaje conocido bajo la denominación inglesa de learning travel que actualmente está transformando la forma tradicional de viajar. [...] Se trata de un nicho de mercado de desarrollo muy prometedor que da lugar a la innovación y a la creatividad”. El valor de los viajes es reafirmado por muchos autores. Cushner (2004) "teacher as traveler / travel as teacher", donde demuestra una necesidad creciente de intermediación por parte del turista (Frochot, Legohérel, 2007). Se trata de un regreso a la función formativa del turismo reivindicada y promovida por los movimientos excursionistas a fines del siglo XIX y principios del XX (Marié, 1982) o en las caravanas 
escolares descritas por Hoibian (2000 b) en la obra de Pociello y Denis (2000) con un nombre evocador: "en la escuela de la aventura, practicas al aire libre la ideología de la conquista del mundo". Un segundo punto común refleja una voluntad declarada de mantener el carácter distintivo del viaje que se puede resumir en "Viajar sí, pero de manera diferente". En esto podemos encontrar fundamentos del modelo "romantic tourist gaze" defendido por Walter $(1982)$ y Urry $(1995,2002)$ en contraposición al “collective tourist gaze”. El turista quiere ser actor de su experiencia turística más que un simple espectador / consumidor. De esta forma, él desarrolla un consumo vivencial (Holbrook, Hirschman, 1982) con una adaptación privativa de los lugares y exige la adaptación de los servicios turísticos a sus expectativas. De hecho, esta personalización que se espera del viaje restringe la masificación del fenómeno, como lo demuestra Walter (1982). Un amplio espacio para los sentidos, las emociones, la estética, las representaciones, el aprendizaje y, por ende, para la experiencia, se traduce, como propone Bousiou (2008), en el desarrollo de una ideología del turismo de "el acto de viajar”. Este último tiene prioridad ante el acto del consumo turístico como tal. La movilidad turística y el consumo están vinculados y se transforman en un medio y no en una finalidad en el desplazamiento. A pesar de que esta observación se aplica a las cuatro formas, permite legitimar el hecho de integrar al fenómeno turístico ciertas movilidades vinculadas a las investigaciones científicas. Desde un punto de vista léxico, a fin de marcar esta diferencia, los proveedores de servicios privilegian frecuentemente el término "viaje" en vez de turismo. El eco-voluntariado habla de "viaje solidario y responsable" para calificar ciertos viajes (www.eco-volontaire.com). En las universidades, se habla de "viajes de estudio”. La expresión “viajes de exploración” permite clasificar el turismo deportivo en los destinos lejanos o exóticos. Esta tendencia está presente en numerosas formas de turismo cultural (Can-Seng, 2002). En estos dos casos, se trata del regreso a los principios fundadores del turismo como el "tour” inscrito en doble proceso distintivo socialmente y formativo.

\section{El turismo científico: forma alternativa y nicho turístico}

Estas particularidades tienden a singularizar o a posicionar el turismo científico en oposición al turismo de masas. El elitismo social y cultural (véase pirámide sociocultural del turismo de Boyer, 1999 a y b) inherente a múltiples nichos turísticos en general, y a las diversas formas de turismo científico en particular, quedará como una limitación estructural y duradera de la masificación del fenómeno. A pesar de que el turismo activo, educativo y voluntario es promovido por diversos operadores y territorios, una de las variables más estables cerca de un 85\% del sondeo del "Suivi des Déplacements Touristiques des Français" (Seguimiento de los Desplazamientos Turísticos de los Franceses), (S.D.T., SOFRES - Observatoire National du Tourisme) muestra que año a año la principal actividad durante los desplazamientos turísticos es “descansar” y “no hacer nada”. Novelli 
(2004) clasifica el "scientific tourism" y el "research tourism" (Benson, 2004) en los nichos turísticos de la misma forma que a los tipos de turismo denominados: deportivo, de aventura, de observación de la fauna salvaje, gastronómica, genealógica, de voluntariado, ética, espacial, etc. Esta diversidad tiende a demostrar una segmentación / hibridación importante de la oferta turística, pero también una recomposición perpetua de la demanda basada en una búsqueda de una experiencia temática y personalizada.

Como todo nicho turístico, el turismo científico se subdivide en subnichos, anteriormente calificados como formas. Estos se pueden diferenciar siguiendo dos ejes (véase figura $\mathrm{n}^{\circ} 1$ ): 1 . El tipo de participación del actor (turista, usuario, diseñador) en la construcción del producto (estadía, actividad, objetivos, expectativas, etc.) por sí mismo; 2. La dimensión científica (lugar de la ciencia en el diseño y el desarrollo de la estadía) de este mismo producto.

Figura $\mathrm{n}^{\circ} 1$. Planteamiento de las diferentes formas de turismo científico

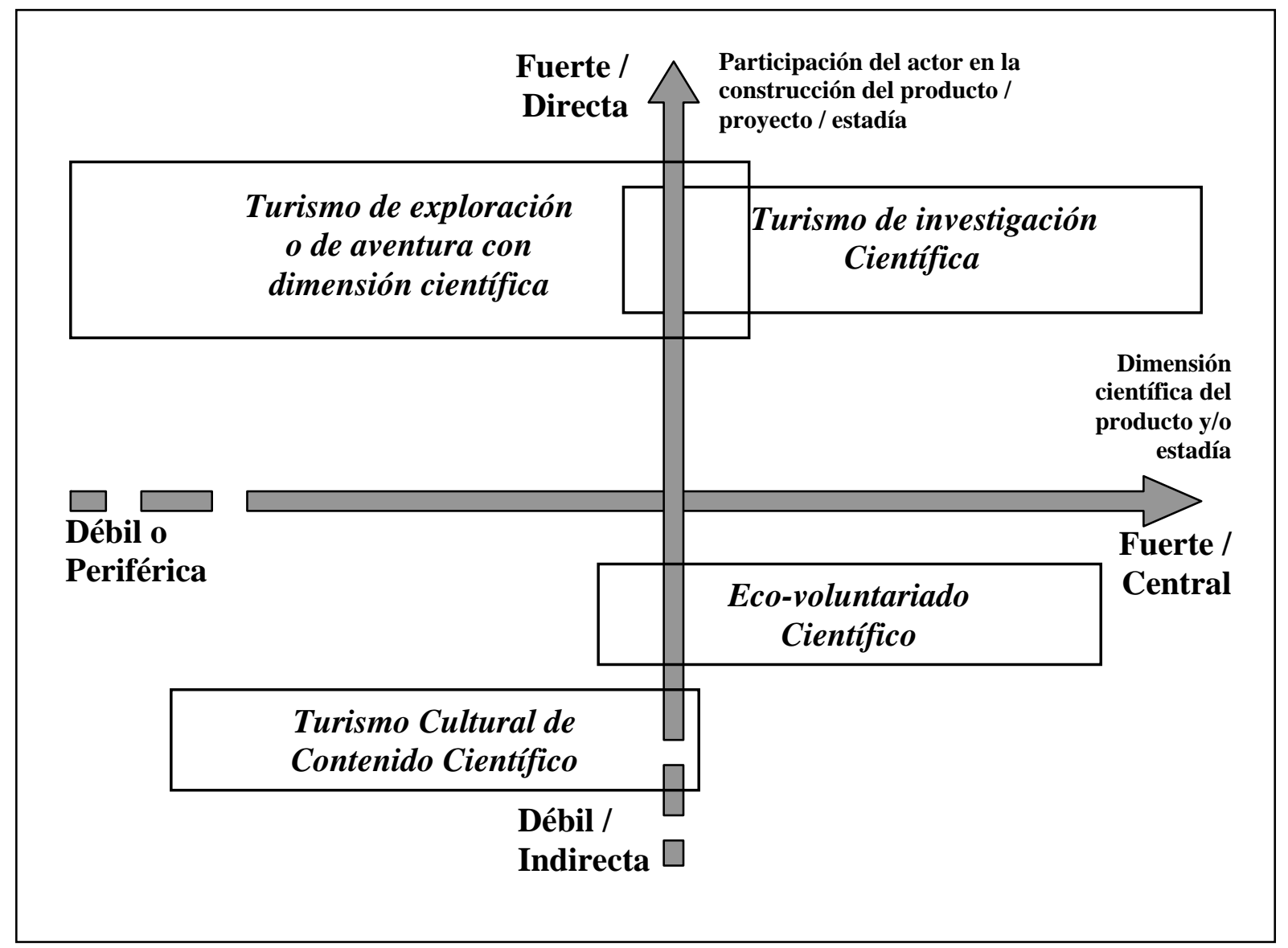

Fuente: Mao y Bourlon, 2011

En el primer eje, en el caso del turismo de aventura de dimensión científica y de investigación científica, los participantes son autoproductores de sus estadías tanto desde el punto de vista de la 
elección del destino como de su organización. Al contrario, el turismo cultural y el ecovoluntariado están inmersos en proyectos o productos preexistentes, y, por lo tanto, pasa por la compra de una prestación comercial frecuentemente personalizada o a pedido. En el segundo eje, el proyecto científico es central en la definición del viaje tanto por las movilidades vinculadas a la investigación como para el ecovoluntario. Al contrario, para el turismo cultural y de aventura, el lugar de la ciencia posee un carácter menos fuerte o central, incluso en algunos casos sólo sirve como pretexto o simple justificación para el desplazamiento turístico. Cabe destacar que en algunos casos de exploración, es difícil identificar qué prevalece de la ciencia o de la aventura (por ejemplo las numerosas expediciones de la National Geographic).

A pesar de que las formas difieren, son frecuentemente complementarias e interdependientes en un mismo destino. Así, las exploraciones deportivas o científicas permiten identificar los recursos naturales y patrimoniales sobre los cuales se realizarán investigaciones posteriores. El ecovoluntariado requiere la puesta en marcha de programas de investigación. La mediación durante las estadías ecoturísticas o culturales imponen un conocimiento científico adquirido previamente y difundido, véase el análisis de Laarman y Perdue (1989), en Costa Rica o de Hall et al. (1992, 1995 et 2010), para los destinos polares.

Cuando aplicamos esta teoría al caso de Aysén observamos una coherencia pero también una complejidad mayor de las formas, principalmente al cruzar dos, tres y hasta cuatro de los segmentos anteriormente definidos. Esto se grafica en esferas (círculos) que se traslapan (véase figura 2). Aparecen entonces 9 sub-formas del turismo Científico: 1. Exploraciones Científicas, 2.Viajes Educativos y Culturales, 3. Exploraciones Culturales y Deportivas, 4. Eco-Voluntariado Científico, 5. Ecoturismo con dimensión Científica 6. Investigaciones Científicas, 7. Exploraciones Deportivas con perspectiva Científica, 8. Viajes Educativos y de aprendizaje y 9 El Viaje de Turismo Científico Integral es el que incorpora de una u otra manera elementos de las 4 formas del turismo científico. 
Figura $n^{\circ} 2$.

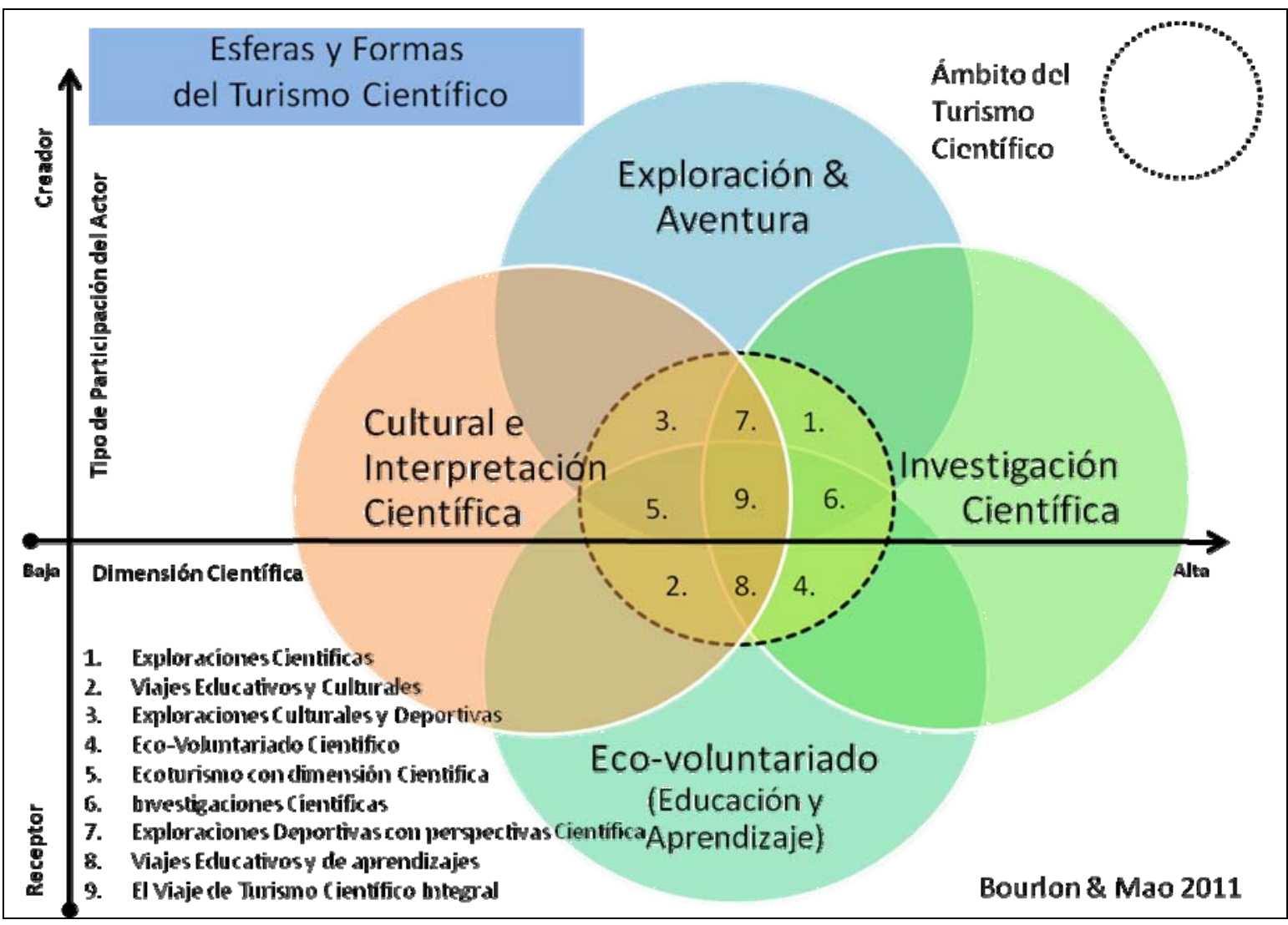

En cada uno de estos segmentos podemos así visualizar un sinfín de posibilidades de productos. Destacamos para Aysén algunos de estos casos, análisis del capítulo 1, y resumido en la tabla $\mathrm{n}^{\circ} 1$. Esta aparente complejidad puede a la vez ser apropiada por los operadores para que sean innovadores en la construcción de nuevos productos. La idea de "territorio turístico creativo" puede aquí encontrar todo su significado. En un espacio turístico "periférico" como Aysén, productos clásicos muestran sus límites lo que obliga a los operadores a una mayor creatividad en su oferta. Si bien el concepto no es nuevo al nivel mundial, en Aysén se está implementando como una estrategia de desarrollo territorial. No se trata solo de una iniciativa de un grupo de actores pero una voluntad política fuerte de basar las dinámicas turísticas en su conjunto en esta perspectiva (cf. www.turismocientifico.cl). 
Tabla $\mathrm{n}^{\circ} 1$

\begin{tabular}{|c|c|c|c|c|}
\hline & $\begin{array}{c}\text { Segmento del } \\
\text { turismo científico }\end{array}$ & $\begin{array}{l}\text { Categorías de } \\
\text { viaje }\end{array}$ & $\begin{array}{l}\text { Temáticas } \\
\text { científicas }\end{array}$ & Nombre del viaje \\
\hline 1 & $\begin{array}{l}\text { Exploraciones } \\
\text { Científicas }\end{array}$ & $\begin{array}{l}\text { Exploración \& } \\
\text { Aventura Científica }\end{array}$ & $\begin{array}{l}\text { Arqueología } \\
\text { Biología de } \\
\text { mamíferos marinos }\end{array}$ & $\begin{array}{l}\text { Exploración arqueológica Valle } \\
\text { Chacabuco, } 2009 \\
\text { Identificación y estudios preliminares } \\
\text { sobre Cetáceos del litoral, 2008-2009. }\end{array}$ \\
\hline 2 & $\begin{array}{l}\text { Viajes Educativos } \\
\text { y Culturales }\end{array}$ & $\begin{array}{l}\text { Cultural e } \\
\text { Interpretación } \\
\text { Científica }\end{array}$ & $\begin{array}{l}\text { Ciencias forestales y } \\
\text { conservación }\end{array}$ & $\begin{array}{l}\text { Circuito. Gestión forestal y } \\
\text { conservación en la RN Coyhaique, } 2011\end{array}$ \\
\hline 3 & $\begin{array}{l}\text { Exploraciones } \\
\text { Culturales y } \\
\text { Deportivas } \\
\end{array}$ & $\begin{array}{l}\text { Exploración \& } \\
\text { Aventura Cultural } \\
\text { y Científica }\end{array}$ & $\begin{array}{l}\text { Geografía e historia } \\
\text { marina }\end{array}$ & $\begin{array}{l}\text { Expedición marítima al sitio de } \\
\text { naufragio de la Fragata Wager en } 2006\end{array}$ \\
\hline 4 & $\begin{array}{l}\text { Eco-Voluntariado } \\
\text { Científico }\end{array}$ & $\begin{array}{l}\text { Eco-voluntariado } \\
\text { (Educación y } \\
\text { Aprendizaje) e } \\
\text { Investigación } \\
\text { Científica }\end{array}$ & $\begin{array}{l}\text { Ciencias } \\
\text { Ambientales y } \\
\text { Ecología de Ríos }\end{array}$ & $\begin{array}{l}\text { Viaje de estudio académico, Gestión } \\
\text { ambiental y ecología del río Baker, Univ. } \\
\text { de Michigan } 2008\end{array}$ \\
\hline 5 & $\begin{array}{l}\text { Ecoturismo con } \\
\text { dimensión } \\
\text { Científica }\end{array}$ & $\begin{array}{l}\text { Cultural e } \\
\text { Interpretación } \\
\text { Científica }\end{array}$ & $\begin{array}{l}\text { Cultura, Medio } \\
\text { Ambiente y } \\
\text { Conflictos Sociales }\end{array}$ & $\begin{array}{l}\text { Viaje de estudio y turismo "Proyectos } \\
\text { hidro-eléctricos en la Patagonia, } \\
\text { encuentros con actores locales. Univ. } \\
\text { Montana } 2011\end{array}$ \\
\hline 6 & $\begin{array}{l}\text { Investigaciones } \\
\text { Científicas }\end{array}$ & & $\begin{array}{l}\text { Arqueología Marina } \\
\text { Biología Marina y } \\
\text { Conductas Animales }\end{array}$ & $\begin{array}{l}\text { Levantamientos científicos en torno a los } \\
\text { sitios del naufragio de la Fragata Wager. } \\
\text { Arkas, UACH CIEP, Mar } 2011 \\
\text { Estudios acústicos y fotométricos de los } \\
\text { cetáceos del litoral de Aysén, Ocean } \\
\text { Sounds - CIEP } 2010\end{array}$ \\
\hline 7 & $\begin{array}{l}\text { Exploraciones } \\
\text { Deportivas con } \\
\text { perspectivas } \\
\text { Científicas }\end{array}$ & $\begin{array}{l}\text { Exploración \& } \\
\text { Aventura }\end{array}$ & $\begin{array}{l}\text { Reconocimientos } \\
\text { Geográficos en el } \\
\text { litoral de Aysén, }\end{array}$ & $\begin{array}{l}\text { Expedición C. Donoso en los fiordos del } \\
\text { Sur de Aysén. } 2006 . \\
\text { Reconocimiento en kayak del litoral } \\
\text { entre Bahía Exploradores y laguna San } \\
\text { Rafael, Pontifica Universidad Católica, } \\
2011 .\end{array}$ \\
\hline 8 & $\begin{array}{l}\text { Viajes Educativos } \\
\text { y de aprendizajes }\end{array}$ & $\begin{array}{l}\text { Eco-voluntariado } \\
\text { (Educación y } \\
\text { Aprendizaje) }\end{array}$ & $\begin{array}{l}\text { Ornitología y } \\
\text { Arqueología }\end{array}$ & $\begin{array}{l}\text { Viaje de observaciones ornitológicas } \\
\text { sobre los cóndores e introducción a la } \\
\text { arqueología en la estancia Punta del } \\
\text { Monte. } 2010\end{array}$ \\
\hline 9 & $\begin{array}{l}\text { El viaje de } \\
\text { Turismo } \\
\text { Científico Integral }\end{array}$ & $\begin{array}{l}\text { Viaje que Incluye } \\
\text { las cuatro formas } \\
\text { del TC: } \\
\text { Investigación, } \\
\text { voluntariado, } \\
\text { aventura y cultura }\end{array}$ & $\begin{array}{l}\text { Estudios Históricos } \\
\text { y Sociales }\end{array}$ & $\begin{array}{l}\text { Programa. Investigación arqueológica en } \\
\text { el Valle Chacabuco Ene. } 2011 \\
\text { Estudios históricos y arqueológicos con } \\
\text { la comunidad rural del Cerro Galera. } \\
2011\end{array}$ \\
\hline
\end{tabular}




\section{A MODO DE CONCLUSIÓN SOBRE EL TURISMO CIENTÍFICO}

Históricamente, los mundos del turismo y de las ciencias han estado fuertemente vinculados. Por consiguiente, la noción de turismo científico que movilizan numerosos autores y operadores a partir de mediados de los años 90, tiene diversos derivados tanto en términos de prácticas como de productos. Este dinamismo ilustra una innovación permanente y una gran creatividad (Corneloup, Mao, 2010) por parte de las formas de turismo denominadas alternativas o de nichos. Desde muchos puntos de vista, estas formas se inscriben en un transturismo (Corneloup, 2009) que apunta a liberarse de los marcos clásicos del turismo moderno e industrial. El desarrollo del turismo científico se apoya, como otros nichos turísticos, en la renovación de las motivaciones turísticas. Se confirma el desarrollo de nichos basados en la ecologización de las prácticas, un aumento de los intermediarios culturales y medioambientales, una investigación existencial y vivencial que da un nuevo sentido o justificación al viaje y, finalmente, a un voluntariado ético y educativo.

Podemos observar en su implementación sistematizada en la región de Aysén como el turismo científico permite innovar y fortalecer un desarrollo turístico diferenciador. Vemos como en este territorio las investigaciones impulsan el turismo y como el turismo puede apoyar el trabajo científico. A través de los ejemplos citados podemos así observar que podemos tener sucesivamente exploraciones que dan pié para investigaciones académicas. Programas de eco-voluntariado que permiten sostener procesos en el tiempo y crear una nueva oferta turística mas cultural y formativa.

\section{BIBLIOGRAFIA}

BAILLET, CAROLINE et ORELIEN BERGE (2009). Comprendre l'expérience de l'éco volontariat : une approche par la valeur de consommation, Actes du Deuxième Colloque International sur les Tendances du Tourisme, Sidi Dhrif : Tunisie.

BELAËN, FLORENCE (2007) Le planétarium: observatoire des tendances, Culture \& musées, 10, pp. 21-36.

BENSON, ANGELA M. (2004), "Research tourism, professional travel for useful discoveries" in Niche tourism: contemporary issues, trends and cases, Oxford: Elsevier, pp. 133-144.

BLANGY, SYLVIE et ALAIN LAURENT (2007) Le tourisme autochtone : un lieu d'expression privilégié pour des formes innovantes de solidarité, Tourisme et solidarité, 26 (3), pp. 38-45. 
BOUCHET, PATRICK y ANNE-MARIE LEBRUN (2009), Management du tourisme sportif, de la consommation à la commercialisation, Rennes: Presses Universitaires de Rennes, $354 \mathrm{p}$.

BOURDEAU, PHILIPPE (2006) La montagne - terrain de jeu et d'enjeux; débats pour l'avenir de l'alpinisme et des sports de nature, Coll. Sportsnature.org, L’Argentière la Bessée : Ed. du Fournel, 207 p.

BOUSIOU, POLA (2008) The nomads of Mykonos: performing liminalities in a 'queer' space, New directions in anthropology, Oxford - New York, Berghahn Series, 308 p.

BOYER, MARC (1999 a), Histoire du tourisme de masse, Coll. Que sais-je ?, Paris: Presses Universitaires de France, $126 \mathrm{p}$.

BOYER, MARC (1999 b), Le tourisme de l'an 2000, Lyon : Presses Universitaires de Lyon, 265 p.

BRETON, JEAN-MARIE (2004), « Paradigme d'écotourisme et sociétés traditionnelles en mutation : le cas de l'outre-mer français », Téoros, 23 (2), pp. 54-60.

BUCKLEY, RALF (2010), Adventure tourism management, Oxford: Butterworth - Heinemann Elsevier, 268 p.

CAN-SENG, OOI (2002), Cultural tourism and tourism cultures: the business of mediating experiences, Copenhagen and Singapore: Copenhagen Business School Press, 277 p.

CAZES, GEORGES (1998), «Tourisme et développement: du modèle intégré au modèle soutenable » in Tourismes, touristes, sociétés, Coll. Tourismes et sociétés, Paris : l’Harmattan, pp. 97-105.

CHRIS, JONES (1997), Climbing in North America, Seattle: Mountaineers Books, 365 p.

CLOT, CHRISTIAN, (2009), 100 ans d'exploration française, La Bibliothèque des explorateurs, Grenoble : Glénat, 367 p.

CORNELOUP, JEAN y PASCAL MAO (2010), Créativité et innovation dans les loisirs sportifs de nature, un autre monde en émergence, Coll. Sportsnature.org, L'Argentière-laBessée : Ed. du Fournel, 415 p.

CORNELOUP, JEAN, (2009), «Comment est abordée la question de l'innovation dans les sciences sociales ? », Revue de Géographie Alpine, 97 (1), pp. 113-128.

COUSIN, SASKIA (1998), «Le tourisme industriel, objet médiatique non identifié », Cahiers Espaces, 57, pp. 6-14

COUSIN, SASKIA (2000), «Un brin de culture, une once d'économie : écomusée et Economusée », Publics et Musées, 17-18, pp. 115-137.

COUSIN, SASKIA (2001), «Industrie de l'évasion ou entreprise d'encadrement ? Le cas du tourisme industriel », Quaderni, 44, pp. 45-71. 
CSL SAGUENEY - LAC ST JEAN (2005), Tourisme scientifique, pour voyager autrement, Comité Régional en Tourisme Scientifique, Sagueney - Lac St Jean, 17 p.

CUSHNER, KENNETH (2004), Beyond tourism: a practical guide to meaningful educational travel, Information and Interdisciplinary Subjects Series, R\&L Education, 165 p.

DEBARBIEUX, BERNARD (2001), «La montagne : un objet géographique ? » in VEYRET (dir.), Les montagnes : discours et enjeux géographiques, Paris : SEDES.

DEWAILLY, JEAN-MICHEL (2006), Tourisme et géographie, entre pérégrinité et chaos? Coll. « Tourismes et sociétés », Paris : L’Harmattan, 221 p.

ELLIS, CLAIRE (2003), "When Volunteers Pay to Take a Trip with Scientists-Participatory Environmental Research Tourism (PERT)”, Human Dimensions of Wildlife, 8, 1, pp. 75-80.

FROCHOT, ISABELLE ET PATRICK LEGOHEREL (2007), Le Marketing du tourisme Paris: Dunod, 275 p.

GONZÁLEZ RODRÍGUEZ, Juan Guillermo (2004), Ecoturismo y pesca deportiva: Parque Nacional

El Tuparro, Universidad Externado de Colombia, Facultad de Administración de Empresas Turísticas y Hoteleras, 211 p.

GRETEL, EHRLICH. (2000), John Muir : nature's visionary, Washington: National Geographic Society, $241 \mathrm{p}$.

GUNN, CLARE A. (1988), Tourism Planning, Washington: Taylor \& Francis, 356p.

GUNN, CLARE A. (1997), Vacationscape: Developing Tourist Areas, Washington: Taylor \& Francis, $196 \mathrm{p}$.

HALL, C MICHAEL (1992), “Tourism in Antarctica: Activities, Impacts and Management”, Journal of Travel Research, 30, pp. 2-9.

HALL, C MICHAEL and MARGARET JOHNSTON (1995), Polar tourism: Tourism in the Arctic and Antarctic regions, Chichester: John, Wiley and Sons Ltd., 346 p.

HALL, C MICHAEL. and JARKKO SAARINEN (2010), "Polar tourism: Definitions and dimensions”, Scandinavian Journal of Hospitality and Tourism, 10(4), pp. 448-467.

HALL, C MICHAEL (2010), “John Muir: pioneer of nature preservation” in Butler and Russell (Ed.), Giants of Tourism, Wallingford: CABI, pp. 229-242.

HOERNER, JEAN MICHEL (2002), Traité de tourismologie. Pour une nouvelle science touristique, Perpignan : Presses de l’Université de Perpignan, 191 p.

HOIBIAN, OLIVIER et JACQUES DEFRANCE. (2002), Deux siècles d'alpinismes européens : origines et mutations des activités de grimpe, Coll. Sports en société Paris: L'Harmattan, 396 p. 
HOIBIAN, OLIVIER (2000 a), Les alpinistes en France, 1870-1950 : une histoire culturelle, Coll. Espaces et temps du sport, Paris : Harmattan, 338 p.

HOIBIAN, OLIVIER (2000 b), « Les caravanes scolaires, un projet pédagogique inédit » in Pociello et Denis, A l'école de l'aventure, pratiques de plein air et idéologie de la conquête du monde, Toulouse, PUS, pp. 45-54

HOLBROOK, MORRIS B. and ELIZABETH C. HIRSCHMAN (1982), "The Experiential Aspects of Consumption: Consumer Fantasies, Feelings, and Fun”, Journal of Consumer Research, 9, pp. 132-140.

HORA, A.S.S. y K.B. CAVALCANTI (2003), “Turismo pedagógico: conversão e reconversão do olhar” in Rejowski y Costa (Orgs.). Turismo Contemporâneo: desenvolvimento, estratégia e gestão, São Paulo: Atlas, pp. 207 - 227.

KNAFOU, REMY et PHILIPPE VIOLIER (2005), «Les mondes urbains du tourisme », EspacesTemps.net, Actuel, 23.06. http://espacestemps.net/document1446.html

LAARMAN, JAN G. and RICHARD R. PERDUE (1989), “Science tourism in Costa Rica”, Annals of Tourism Research, 16 (2), pp. 205-215.

LAMIC, JEAN-PIERRE (2008), Tourisme durable: utopie ou réalité? : comment identifier les voyageurs et voyagistes éco-responsables, Coll. Tourismes et sociétés, Paris : 219 p.

LEIPER, NEIL (1995), Tourism Management, Melbourne: RMIT Press, 367p.

LEVI-STRAUSS, CLAUDE (1955), Tristes Tropiques, Paris : Plon, 462 p.

MALECOT, YVES (1981) La Culture scientifique, technique et industrielle, Paris: la Documentation française.

MARIE, MICHEL (1982), Un territoire sans nom, pour une approche des sociétés locales, Coll. Sociologie au quotidien, Paris : Librairie des Méridiens, 180p.

MICHEL, FRANK (1998), « Des manières d'être et de faire du tourisme et de l'anthropologie, une rencontre impossible et/ou impensable " in Tourismes, touristes, sociétés, Coll. Tourismes et sociétés, Paris : l’Harmattan, pp. 97-105.

MIECZKOWSKI, ZBIGNIEW (1995) Environmental Issues of Tourism and Recreation, Maryland: University Press of America, 566p.

MILLER, SALLY M. and DARYL MORRISON (2005), John Muir: family, friends, and adventures, Albuquerque: UNM Press, $281 \mathrm{p}$.

MINISTÉRIO DO TURISMO (2010) Turismo de estudos e intercâmbio: Orientações Básicas, Brasília: Secretaria Nacional de Políticas de Turismo, 75p.

MIT (2002), Tourisme 1. Lieux communs, Paris : Belin, 320 p.

MORSE, MICHAEL A. (1997), “All the world's a field: a history of the scientific study tour”, Progress in Tourism and Hospitality Research, 3 (3), pp. 257-269. 
MUIR, JOHN (1992), John Muir: the eight wilderness discovery books, Seattle: The Mountaineers Books, 1030 p.

NOVELLI, MARINA (2004) Niche tourism: contemporary issues, trends and cases, Oxford: Elsevier, 288p.

ORIGET DU CLUZEAU, CLAUDE (2005), «Tourisme scientifique et tourisme culturel destins communs et divergences ", La lettre de l'OCIM, 101, pp. 6-11.

PAUCHARD, ANÍBAL (2000), "La experiencia de Costa Rica en áreas protegidas”, Ambiente y Desarrollo, Vol. XVI - 3, pp. $51-60$.

POCIELLO, CHRISTIAN et DANIEL DENIS (2000), A l'école de l'aventure, pratiques de plein air et idéologie de la conquête du monde, Toulouse : Presse Universitaire du Sport, 325 p.

ROVINSKY, YANINA (1993) "Private Reserves, Parks and Ecotourism in Costa Rica” in Nature Tourism: Managing for the environment, Washington: USA.

SELTERS, ANDY (2004), Ways to the sky: a historical guide to North American mountaineering, American Alpine book series, Seattle: The Mountaineers Books, 334 p.

SHAW, GARETH, and ALLAN M. WILLIAMS (2002), Critical issues in tourism: a geographical perspective, IBG Studies in geography,Wiley-Blackwell, 371 p.

SIMMONS, DAVID and NEIL LEIPER (1993), “Tourism: A Social Science Perspective” in: Harvey C. Perkins and Grant Cushman (eds), Leisure, recreation and tourism, Auckland: Longman Paul.

STEBBINS, ROBERT A. and MARGARET GRAHAM., (2004), Volunteering as leisure/leisure as volunteering: an international assessment, Wallingford : CABI Publishing Series, 253 p.

STOCK, MATHIS (2003), Le tourisme. Acteurs, lieux et enjeux, Paris : Belin, $304 \mathrm{p}$.

THURNER, INGRID (1999), “Wissenschaftstourismus: Der Forscher als Tourist?”, Mitteilungen der Anthropologischen Gesellschaft in Wien, 129, pp. 227-246.

URRY, JOHN (1995), Consuming places, Londres: Routledge, 257 p.

URRY, JOHN, (2002), The tourist gaze, Theory, culture \& society, Published in association with Theory, Culture \& Society, SAGE, 183 p.

VARGAS ULATE, GILBERT (1997), Geografía turística de Costa Rica, San José: EUNED, 180 p. WALTER, TONY (1982), “Social Limits to Tourism”, Leisure Studies, 1 (3), pp. 295-304.

WEARING, STEPHEN (2001), Volunter tourism: experiences that make a difference, Wallingford: CABI. Publishing, $240 \mathrm{p}$.

WEARING, STEPHEN and JOHN NEIL (2000), "Refiguring self and identity through volunteer tourism”, Loisir et société, 23 (2), pp. 389-419. 
WILKIE, DAVID S. and JULIA CARPENTER (1999) “Can nature tourism help finance protected areas in the Congo Basin?”, Oryx, 33 (4), pp.330-339.

Especial agradecimiento a Francisco Carmona por los aportes en cuanto a la traducción y revisión del francés al español.

Recibido: 06/06/2011

Aprobado: 30/06/2011

Arbitrado anónimamente 